\title{
Highlights of the issue 5, 2020
}

Published online: 27 April 2020

(C) Fondazione Società Italiana di Neurologia 2020

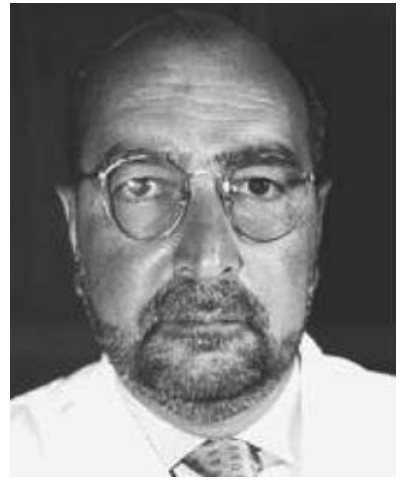

Antonio Federico

Editor-in-Chief

\section{COVID-19}

Acute stroke management pathway during Coronavirus-19 pandemic.

Claudio Baracchini1 \& Alessio Pieroni1 \& Federica Viaro1 \& Vito Cianci2 \& Anna M. Cattelan3 \& Ivo Tiberio4 \& Marina Munari5 \& Francesco Causin6

(Italy)

https:/link.springer.com/article/10.1007/s10072-020-04375-9

Since the outbreak of the COVID-19 epidemic which in our region, Veneto (Italy), dates back to February, we were confronted with several challenges, but with a constant aim of keeping our Stroke Unit COVID-free. For this reason, in addition to creating a dedicated hot-spot as a pre-triage just outside the Emergency Department, together with the Neuroradiology Unit we obtained a mobile CT unit that could be used by COVIDpositive or COVID-suspected patients. Furthermore, thanks to the collaboration with colleagues from different specialties (Infectious Disease, Internal Medicine, Intensive Care, Emergency Medicine), dedicated areas for COVID patients were activated. This led to a substantial change of our acute stoke management pathway. As the number of COVID patients increased, and the WHO declared a state of pandemic, this new stroke pathway has been fully tested. We would like to share our experience and send a clear message to keep a high attention on stroke as an emergency condition, because we have observed a decreased number of patients with minor strokes and TIAs, longer onset-to-door and door-to treatment times for major strokes, and a reduced number of transfers from spokes. We strongly believe that the general population and family doctors are rightly focused on COVID. However, to remain at home with stroke symptoms does not mean to "stay safe at home".

Digital triage for people with multiple sclerosis in the age of COVID-19 pandemic

Simona Bonavita1 \& Gioacchino Tedeschi1 \& Ashish Atreja2 \& Luigi Lavorgna1

(Italy)

https:// link.springer.com/article /https://doi.org/10.1007/ s10072-020-04391-9

We propose a possible approach for the remote monitoring of infection risk in people with multiple sclerosis, especially those on immunosuppressant drugs, during COVID-19 pandemic. We developed a digital triage tool to be sent to patients to quickly identify people with high risk of COVID-19 infection. This tool will also limit unnecessary accesses to the MS centers reducing the risk of spreading the infection.

\section{REVIEW ARTICLES}

Recent progress in neuronal intranuclear inclusion disease: a review of the literature

HafizKhuramRaza1 \& SandeepSingh2 \& PabitraRai2 \& Thitsavanh Chansysouphanthong2 \& AroojAmir3 \& GuiyunCuil \& Wenjing Song1 \& Lei Bao1 \& Su Zhou1 \& Hongjuan Shil \& Hao Chen1

(China, Pakistan)

https://ink.springer.com/article/10.1007/s10072-019-04195-6 
Objective To summarize the current understanding of neuronal intranuclear inclusion disease (NIID) and improve the understanding of the physician about this condition. Methods The AA searched PubMed with keywords related to NIID and selected publications which seemed appropriate. They analyzed its clinical features, pathogenesis, evaluation methods, treatment options, and research perspectives. Results NIID is a degenerative condition which can affect multiple organ systems especially central nervous system. Its clinical features greatly vary, and making the exact diagnosis is often difficult. There are several genes which have been associated with this disorder. Some specific signs on diffusion-weighted-imaging (DWI) sequence of magnetic resonance (MR) imaging are characteristics to NIID. Conclusion Intranuclear inclusions have been found in various non neural cells of the body; therefore, the term systemic intranuclear inclusion disease is, perhaps, better suited to explain this disorder. There are several disorders which need to be ruled out before making the diagnosis, and neuroimaging and biopsy analysis should be combined to support the diagnosis.

\section{Theory of mind in Alzheimer's disease and amnestic mild cognitive impairment: a meta-analysis}

ZhongQuan Yi1 \& PanWen Zhao1 \& Hui Zhang1 \& YuanYuan Shi1 \& HaiChun Shi2 \& JianGuo Zhong2 \& PingLei Pan1,2

(China)

https://ink.springer.com/article/10.1007/s10072-019-04215-5

Background The assessment of theory of mind (ToM) performance in Alzheimer's disease (AD) and amnestic mild cognitive impairment (aMCI) remains inconclusive. The conducted a meta-analysis to investigate ToM performance in patients with aMCI and AD. Methods A systematic literature search was performed for eligible studies published up to July 2019 in three international databases (PubMed, Embase, and Web of Science). Due to heterogeneity between studies, a randomeffects model was used. Individual ToM tasks were metaanalyzed separately and possible sources of heterogeneity were examined. Results In total, 36 studies involving 701 individuals with $\mathrm{AD}$ and 197 with aMCI were identified. Compared with healthy controls, ToM was impaired in both $\mathrm{AD}(\mathrm{d}=1.45)$ and aMCI patients $(\mathrm{d}=0.65)$. In AD patients, ToM was particularly impaired in advanced tasks such as Faux Pas Recognition $(\mathrm{d}=1.26)$. In patients with aMCI, ToM deficits were relatively modest, with the exception of the reading the mind in the eyes task $(\mathrm{d}=1.22)$. ToM was significantly more impaired in AD than that in aMCI $(\mathrm{d}=$ 0.88). Conclusions This is the first meta-analysis examining ToM performance in $\mathrm{AD}$ and aMCI simultaneously. The results showed that ToM deficits were more severe in $\mathrm{AD}$ than that in aMCI in most individual ToM tasks. Longitudinal studies are warranted to determine whether ToM abilities in aMCI patients can be used for prognostic purposes.

The associations of HLA-DRB1 gene polymorphisms with late-onset myasthenia gravis: a meta-analysis

Cai-Se Ling1,2 \& Ming-Li Shen1 \& Yi Wang3 \& Wen-Ke Cai4 \& Xiao-Qian Lin5 \& Qian Huang1 \& Gong-Hao He1 (China)

https://ink.springer.com/article/10.1007/s10072-019-04213-7

Background Late-onset myasthenia gravis (LOMG) is one of the major subgroups of the MG. Intensive evidence suggested that polymorphisms in HLA-DRB1 gene were associated with LOMG risk, but the results remained inconsistent. Therefore, a metaanalysis is conducted to make a more precise evaluation between HLA-DRB1 alleles and LOMG. Methods The PubMed, EMBASE, Cochrane library, Chinese National Knowledge Infrastructure (CNKI), and Wan Fang and Technology of Chongqing (VIP) Database were searched for eligible studies. The pooled odds ratios (ORs) and corresponding $95 \%$ confidence intervals (CIs) were applied to assess the association between HLA-DRB1 alleles and LOMG. Results A total of 11 studies involving 5513 people were included in our meta-analysis. The results showed that DRB1 07 and 0403 alleles were risk factors for LOMG $(1.83$ [1.12,2.98], $P=0.02$; 7.05[2.62,18.92], $P=0.0001$, respectively), while DRB1 0301 and 1301 alleles were identified as protective factors for LOMG (0.44 [0.31, 0.62], $P<0.00001 ; 0.38$ [0.23, 0.62], $\mathrm{P}=0.0001$, respectively). As for the HLA-DRB1 04 and 14 alleles, our subgroup analysis showed that there were significant associations between these alleles and LOMG in Caucasians (2.21 [1.14, 4.27], $\mathrm{P}=0.02 ; 2.82$ [1.29, 6.14], $P=0.009$, respectively). Conclusions These results confirmed the association of DRB1 alleles (0301, 04, 0403, 07, 1301, and 14) and LOMG, which might provide potential promising biomarkers for prediction of LOMG risk.

The role of noninvasive brain stimulation for behavioral and psychological symptoms of dementia: a systematic review and meta-analysis

Xin Wang1,2 \& Zhiqi Mao2 \& Xinguang Yu1,2 (China)

https://ink.springer.com/article/10.1007/s10072-020-04245-4

Objective This meta-analysis aimed at evaluating and comparing the efficacy of noninvasive brain stimulation (NIBS) techniques on the behavioral and psychological symptoms of 
dementia (BPSD). Methods An exhaustive literature retrieval was performed on PubMed, Embase, Cochrane Library, and Web of Science until October 2019. The primary outcome was the relative changes in BPSD severity scores immediately after NIBS and at the last follow-up visit. Subgroup analyses were conducted to compare the efficacy of repetitive transcranial magnetic stimulation (rTMS) and transcranial direct current stimulation (tDCS). Changes in the severity scores after NIBS were also analyzed with restriction to patients with Alzheimer's disease (AD). Results Ten studies with 324 patients were included, out of which 7 studies involved patients with AD. The analysis results indicated that NIBS significantly improved the BPSD outcome immediately after stimulation (SMD, 0.31; 95\% CI, 0.10-0.52; $P=0.005$ ), but not at the last follow-up visit $(0.15 ;-0.11-0.41 ; 0.25)$. The subgroup analyses suggested that the favorable effects of rTMS remained significant at the last follow-up visit $(0.57 ; 0.18-0.96$; $0.004)$. This discrepancy maybe caused by the continuously insignificant outcomes of tDCS on the whole data. The results for $\mathrm{AD}$ patients immediately after stimulation $(0.37 ; 0.12-$ $0.61 ; 0.003)$ and at the last follow-up visit $(0.29 ;-0.19-0.76$; 0.24 ) were both largely similar to those in the whole patient group with dementia. Conclusions rTMS, rather than tDCS, was capable of persistently improving the BPSD at an early stage after treatment. More trials are warranted to confirm our results before the establishment of final conclusions.

\section{ORIGINAL ARTICLES}

\section{Eye movement abnormalities are associated with brainstem atrophy in Wilson disease}

JaromírHanuška1,2 \& PetrDušek1,3 \& JanRusz1,4 \& OlgaUlmanová1 \& AndreaBurgetová3 \& EvženRůžička1 (Czech Republic)

https:/link.springer.com/article/10.1007/s10072-019-04225-3

Backgrounds This study aims to characterize eye movement abnormalities in Wilson disease and examine their association with the degree of brainstem atrophy. Methods Twenty patients (10 males, mean age 46.8, SD 8.9 years) with genetically confirmed neurological WD on stable anticopper treatment and 20 age- and sex-matched healthy subjects were examined. Eye movements, including prosaccade and antisaccade tasks, were evaluated using infrared videooculography. MRI was performed using 1.5 T system, and T2-weighted images were used for the measurement of midbrain and pontine area on midsagittal slices. Clinical severity was assessed using the Unified Wilson's Disease Rating Scale (UWDRS). Results Compared to healthy controls, WD patients showed prolonged latencies of horizontal prosaccades and hypometry of both horizontal $(p=$
$0.04)$ and vertical $(p=0.0046)$ prosaccades. In the antisaccade task, WD patients showed prolonged latency of both horizontal $(\mathrm{p}=0.04)$ and vertical antisaccades $(p=0.047)$ and increased error rate of vertical antisaccades $(p=0.04)$. There is a significant association between midbrain area and horizontal latencies $(\mathrm{r}=-0.53 ; p=0.02)$ and vertical maximum speed in prosaccades $(r=0.47 ; p=0.04)$. The pons area inversely correlated with horizontal prosaccade and antisaccade latencies ( $p=$ 0.007). Conclusions The AA showed impairments of ocular saccades such as prolonged latencies, hypometry, and increased error rate in antisaccades. The strong association between prolonged latencies of prosaccades and the brainstem atrophy suggests that VOG might serve as a sensitive electrophysiological marker of brainstem dysfunction in WD.

Validation of the Freezing of Gait Questionnaire in patients with Parkinson's disease treated with deep brain stimulation

Ota Gal1 \& Kamila Polakova1 \& Hana Brozova1 \& Ondrej Bezdicek1 \& Martina Hoskovcova1 \& Robert Jech1 \& Evzen Ruzicka1

(Czech Republic)

https://ink.springer.com/article/10.1007/s10072-019-04209-3

Background The Freezing of Gait Questionnaire (FoG-Q) is a fast and sensitive assessment tool for freezing (FoG). Objective The objective of the study is for validation of a Czech version of FoG-Q. A further, explorative aim was to examine what FoG-Q indicates about the presence and severity of gait impairment in patients treated with DBS in their full OFF state. Design The study was a cross-sectional validation study. Methods The AA translated FoG-Q following standardized validation protocol. They assessed 35 patients with PD and STNDBS using history taking, UPDRS, Hoehn and Yahr staging, Mini Mental State Examination, Frontal Assessment Battery, FoG-Q, Short Falls Efficacy Scale International, and Beck Depression Inventory, Second Edition. UPDRS III, clinical and instrumental gait assessment, was repeated OFF $\mathrm{MED} / \mathrm{dB} \mathrm{OFF}$ and OFF MED/dB ON. Results Internal consistency of FoG-Q was excellent $(\alpha=0.91)$ as well as convergent (significant correlations with UPDRS II item 14, UPDRS III item 29, several TUG parameters, and FoG Score) and divergent validity (no association with UPDRS I). OFF $\mathrm{MED} / \mathrm{dB}$ OFF, the total FoG-Q score correlated with UPDRS III items 29, 30, and PIGD subscore, step time variability, and negatively with step length and velocity. Limitations Limitation of the study is a relatively small sample size. In conclusion, the Czech translation of FoG-Q is valid. With respect to gait and balance, FoG-Q does, to a certain extent, reflect the native state of the disease in patients treated with high frequency STN DBS. 
The Visual Scanning Test: a newly developed neuropsychological tool to assess and target rehabilitation of extrapersonal visual unilateral spatial neglect

Marco Borsotti1 \& Irene Eleonora Mosca1 \& Francesca Di Lauro2 \& Silvia Pancani1 \& Cristiano Bracali2 \& Tomas Dore3 \& Claudio Macchil \& Francesca Cecchi1 \& the IRCCS Don Gnocchi Stroke Group

(Italy)

https://ink.springer.com/article/10.1007/s10072-019-04218-2 Purpose Visual unilateral spatial neglect (VUSN) is a neuropsychological condition commonly experienced after stroke whereby patients are unable to attend to stimuli on the controlesional side of their space. VUSN can occur in the personal, peripersonal, and/or extrapersonal portion of patient's space. Traditional paper-and-pencil neuropsychological tests are widely used to evaluate VUSN, but they assess peripersonal VUSN. Instead, personal and extrapersonal neglect are less easily evaluated. The aim of this study was to present normative values for the VisualScanningTest(VST), a new neuropsychological tool to quantitatively assess the extrapersonal VUSN. Methods Eighty-six healthy subjects took part in the study (61female), with a mean age of 52.8 years $(\mathrm{SD}=17.0)$ and a mean of 14.0 years of education $(\mathrm{SD}=5.2)$. The VST involved a visual search for a target between similar visual distractors, projected in the far space. The test was administered twice to each participant, with an interval of 2 weeks. From the recorded data, it was possible to obtain indexes related to the reaction times and to the accuracy of the performance on the VST. Results Multiple linear regression analysis revealed that age and education significantly influenced VST-derived indexes. From the regression analysis, a correction grid for raw scores was built. Adjusted scores were then ranked, and by means of a nonparametric procedure, tolerance limits (both outer and inner one-sided) were defined. Conclusions The present study provided normative data for the VST in an Italian population useful for both clinical and research purposes.

The relationship between obstructive sleep apnea and Parkinson's disease: a systematic review and meta-analysis

A-Ping Sun1 \& Na Liu1 \& Ying-Shuang Zhang1 \& Hai-Yan Zhaol \& Xiao-Lu Liul

(China)

https://link.springer.com/article/10.1007/s10072-019-04211-9

Background Obstructive sleep apnea (OSA) is a common sleep disorder in Parkinson's disease (PD). However, the relationship between OSA and PD is still inconsistent. This study was aimed to evaluate the relationship between PD and OSA. Methods Studies on OSA and PD were searched using PubMed, Embase, Web of Science, Cochrane library, and Chinese National Knowledge Infrastructure databases. Review Manager 5.3 software was used to calculate the pooled estimate effect. The inverse variance model was used to pool the mean difference (MD) or hazard ratios (HRs); the Mantel-Haenszel method was used to pool the odds ratio (OR). Heterogeneity among the studies was assessed using I2 statistic and Q test. Results A total of 12 studies with 93,332cases were deemed eligible and included in our metaanalysis. Overall, the occurrence of PD was more frequent in patients with OSA (HR 1.59, 95\% CI, 1.36-1.85). The subgroup analysis demonstrated the risk similarly by sex. Male and female had HR of incident PD with OSA of 1.56 (95\%CI,1.30-1.87) and 1.60 (95\%CI,1.21-2.11), respectively. The incidence of OSA did not increase in PD patients (OR $0.89,95 \% \mathrm{CI}, 0.53-1.49)$. The MD of apnea-hypopnea index (AHI) in PD patients was also not statistically significant $(P=$ $0.5)$. Conclusions The results indicate that OSA is one of independent risk factors of PD. However, OSA does not seem to be abnormally frequent in PD.

\section{The frequency of central nervous system complications in the Cypriot cohort of ATTRV30M neuropathy transplanted patients}

Efthimios Dardiotis1,2 \& Savanna Andreou1 \& Athina-Maria Aloizou2 \& Elena Panayiotou1 \& Vasileios Siokas2 \& MihaelaNedeaIoannou3 \& EmmeliaVounou3 \& KyproulaChristodoulou4 \& GeorgeA.Tanteles5 \& DemosMichaelides6 \& Theodoros Kyriakides1,7 (Cyprus, Greece)

https://ink.springer.com/article/10.1007/s10072-019-04176-9

Background Hereditary transthyretin amyloidosis (ATTR) is a hereditary, sensorimotor and autonomic neuropathy caused by deposits of mutated transthyretin (TTR). The commonest TTR mutation is V30M (ATTRV30M) with patients usually living for about 10 years after disease onset. Liver transplantation (LT) until recently was considered the standard treatment. Objective and methods This study aims to assess the frequency of CNS complications in post-LT patients from the Cypriot cohort. Epidemiological data were collected for all genetically confirmed ATTRV30M neuropathy patients diagnosed at CING since 1992, and CNS-associated symptoms were assessed and evaluated by two neurology specialists. Results Out of the 48 transplanted patients, 10(20.8\%)presented with a CNS complication. All patients had ocular involvement, mainly glaucoma (7/10). Eight presented with transient focal neurological episodes (TFNEs), with expressive dysphasia being reported by four of them. The mean time of TFNE- 
emergence was 16.6 years after the LT. Three died from cerebral hemorrhage. Conclusions CNS complications in post-LT ATTRV30M patients are not rare and usually manifest themselves at a time that surpasses the mean time the patients would have survived without a LT. CNS involvement is associated with increased mortality, due to cerebral hemorrhage.

\section{Efficacy and safety of cerebrolysin in neurorecovery after moderate-severe traumatic brain injury: results from the CAPTAIN II trial}

Dafin F Muresanu1,2 \& Stefan Florian1 \& Volker Hömberg3 \& Christian Matula4 \& Nicole von Steinbüchel5 \& Pieter E Vos6 \& Klaus von Wild7 \& Codruta Birle1,2 \& Ioana Muresanu1,2 \& Dana Slavoaca1,2 \& Olivia Verisezan Rosu1,2 \& Stefan Strilciuc1,2 \& Johannes Vester8 (Romania, Germany, the Netherlands)

https://link.springer.com/article/10.1007/s10072-019-04181-y

Introduction The objective of this trial was to evaluate the efficacy and safety of Cerebrolysin in treating patients after moderate to severe traumatic brain injury (TBI) as an adjunct to standard care protocols. The trial was designed to investigate the clinical effects of Cerebrolysin in the acute (neuroprotective) stage and during early and long-term recovery as part of a neurorestorative strategy. Materials and methods The study was a phase IIIb/IV single-center, prospective, randomized, double-blind, placebo-controlled clinical trial. Eligible patients with a Glasgow Coma Score (GCS) between 7 and 12 received study medication $(50 \mathrm{ml}$ of Cerebrolysin or physiological saline solution per day for 10 days, followed by two additional treatment cycles with $10 \mathrm{ml}$ per day for 10 days) in addition to standard care. We tested ensembles of efficacy criteria for 90, 30, and 10 days after TBI with a priori ordered hypotheses using a multivariate, directional test, to reflect the global status of patients after TBI. Results The study enrolled 142 patients, of which 139 underwent formal analysis (mean age $=47.4$, mean admission GCS $=10.4$, and mean Baseline Prognostic Risk Score $=2.6$ ). The primary endpoint, a multidimensional ensemble of 13 outcome scales, indicated a "smallto-medium"-sized effect in favor of Cerebrolysin, statistically significant at day 90 (MW combined $=0.59,95 \%$ CI 0.52 to $0.66, P=0.0119)$. Safety and tolerability observations were comparable between treatment groups. Conclusion Our trial confirms previous beneficial effects of the multimodal, biological agent Cerebrolysin for overall outcome after moderate to severe TBI, as measured by a multidimensional 1 approach. Study findings must be appraised and aggregated in conjunction with existing literature, as to improve the overall level of insight regarding therapeutic options for TBI patients. The widely used pharmacologic intervention may benefit from a large-scale observational study to map its use and to establish comparative effectiveness in real-world clinical settings.

Utility of a scoring system for differentiating cancerassociated stroke from cryptogenic stroke in patients with cancer

Yuzo Hasegawa1 \& Taiki Setoguchi1 \& Tsukasa Sakaida1 \& Toshihiko Iuchi1

(Japan)

https://ink.springer.com/article/10.1007/s10072-019-04231-5

Background The commonest type of ischemic cerebral stroke in patients with cancer is cryptogenic stroke(CRS), the majority of which are considered cancer-associated strokes (CAS) caused by multiple microemboli associated with hypercoagulation, known as Trousseau syndrome. However, detection of microemboli and diagnosing CAS is difficult. We have therefore developed a scoring system for diagnosing CAS. Methods The AA retrospectively examined data of patients with cancer and stroke between 2006 and 2017. They identified risk factors for CRS, assigned them one or two points, and calculated total scores (Trousseau score) for each patient. They used overall survival after stroke (OSs) to validate the utility of the system. Results In181 consecutive strokes, CRS was the commonest type $(43.6 \%)$ and had a short OSs (median 56 days). They identified the following five risks for CRS: highD-dimer concentration $(\geq 10.0 \mu \mathrm{g} / \mathrm{mL})$ and lesions in multiple territories (two point seach); and active cancer, low platelet count $(150,000 / \mu \mathrm{L}>)$ and female sex (one point each). Trousseau score $\geq 3$ indicated CAS (50.3\%), which had a median OSs of 50 days. Only CAS (hazard ratio 3.44 [2.34 5.10], $P<0.0001)$ and poor performance status ( 3 or 4$)(2.27$ [1.50-3.39], $P=0.0002$ ) were risk factors for OSs; $\mathrm{CRS}$ was not. OSs of patients with non-CAS/CRS was significantly longer than that of those with CAS/CRS (404.5 days vs. 47 days, $P=$ 0.0114), whereas OSs of CAS/non-CRS was much shorter than that of non-CAS/non-CRS (53 days vs. 547 days, $\mathrm{P}<0.0001$ ). Conclusion Trousseau scores simply and clearly identify CAS.

\section{Premorbid functional reserve modulates the effect of re- habilitation in multiple sclerosis}

Letizia Castelli1 \& Laura De Giglio2,3 \& Shalom Haggiag4 \& Arianna Traini5 \& Francesca De Luca6 \& Serena Ruggieri4 \& Luca Prosperini4

(Italy)

https://link.springer.com/article/10.1007/s10072-019-04237-z

Background Premorbid physically and intellectually enriching lifestyles have increasingly been recognized as able to mitigate the risk of disease-related disability in patients with 
multiple sclerosis (MS). Objective To explore if premorbid physical activity, cognitive reserve and trait personality act as proxies for functional reserve that contributes to rehabilitation outcome. Methods We recruited all patients previously enrolled in two pilot trials investigating the effect of homebased video game training in improving balance (Study 1) and attention (Study 2) for additional assessments with the Historical Leisure Activity Questionnaire (HLAQ; a proxy for premorbid physical activity), Cognitive Reserve Index Questionnaire (CRIQ), and Temperament and Character Inventory (TCI). Hierarchical logistic regression (HLR) analyses tested the association of HLAQ, CRIQ, and TCI with training effect on balance (static posturography) and on attention (Symbol Digit Modalities Test). Results The AA identified $94 \%$ (34/36) and 74\% (26/35) of patients participating at the original Study 1 and Study 2, respectively. HLR analyses showed an exclusive "intra-modal" modulation of rehabilitation outcome by functional reserve, given that (1) larger training effect on balance was associated with higher HLAQ $(\mathrm{OR}=2.03, p=0.031)$; (2) larger training effect on attention was associated with higher CRIQ (OR $=1.27, p=0.033$ ). Furthermore, they found specific personality traits associated with (1) greater training effect on balance (self-directedness; $\mathrm{OR}=1.40, p=0.051$ ) and lower training effect on attention (harm avoidance; $\mathrm{OR}=0.66, p=0.075$ ). Conclusion The AA hypothesize that premorbid physical and intellectual activities not only act as a buffer for limiting the MS-related damage but also as functional reserve that can be retrieved by taskoriented training to promote recovery through rehabilitation.

\section{Neuropsychological assessment in acute stroke patients}

Fabrizio Pasotti1,2 \& Francesca G. Magnani3,4 \& Marcello Gallucci5 \& Gerardo Salvato2,4 \& Daniela Ovadia2 \& Massimiliano Scotto3 \& Stefano Merolla6 \& Sandro Beretta6 \& Giuseppe R. Micieli7 \& Elio C. Agostoni8 \& Giovanna Beretta9 \& Gabriella Bottini2,3,4 (Italy

https://ink.springer.com/article/10.1007/s10072-019-04236-0

Background and purpose The number of people suffering from stroke is strongly increasing, giving rise to multiple cognitive deficits which frequently prevent a full recovery. The identification of both spared and impaired cognitive domains has a key role to plan adequate interventions. However, the existing standard tests are either too expensive in terms of time and efforts for patients in acute stage or they derived from instruments addressing different pathologies such as dementia. Methods The AA developed a brief neuropsychological battery (mental performance in acute stroke, MEPS) to assess different cognitive domains (language, memory, praxis, visual perception) in acute stroke patients. MEPS was validated by enrolling a sample of 204 patients suffering from stroke in acute stage, and 263 healthy controls participants. Results The results indicated an adequate construct validity and a high ability in discriminating patients from healthy controls. Conclusions MEPS can be considered a simple and highly valuable bed side battery, easy to administer, with values of sensitivity and specificity suitable to be proposed as a screening tool for patients with acute stroke.

\section{Epilepsy over the centuries: a disease survived at the time}

\author{
Domenico Chirchiglia1 \& Pasquale Chirchiglia1 \\ Italy
}

https://link.springer.com/article/10.1007/s10072-019-04214-6

In this study, the AA have made a historical review of epilepsy through the centuries, from pre-Christian era to the present time. The epileptic was examined by Hippocrates, the first to recognize epilepsy as a disease and not as a supernatural manifestation called Morbus Sacer. The dark years of the Middle Ages were the worst for the disease, where the sufferer was even subjected to torture. The period of the Renaissance saw the epileptic isolated from society while the Enlightenment century improved the knowledge about the disease, thanks also to the first autopsies that showed post traumatic neurological lesions. However, some stigmata of the disease that prevented the marriage of epileptics persisted. It was the prelude to the years 1800-1900, characterized by Lombrosian concepts and Nazi convictions. Lombroso included epileptics among delinquents and criminals, identifying them as such on the basis of physical alterations. Nazi racism introduced the concept of eugenics excluding the epileptic from the so-called pure race. Today, epilepsy is considered a treatable neurological disorder. Morbus Sacer belongs to a remote past. 